\title{
Near-saddle-point-energy photoionization microscopy images of Stark states of the magnesium atom
}

\author{
P. Kalaitzis, ${ }^{1}$ S. Danakas, ${ }^{1}$ C. Bordas, ${ }^{2}$ and S. Cohen ${ }^{1, *}$ \\ ${ }^{1}$ Atomic and Molecular Physics Laboratory, Physics Department, University of Ioannina, 45110 Ioannina, Greece \\ ${ }^{2}$ Université de Lyon, CNRS, UMR5306, Institut Lumière Matière, 69622 Villeurbanne, France
}

(Received 20 December 2018; published 26 February 2019)

\begin{abstract}
Photoionization microscopy (PM) is a photoelectron imaging method based on the measurement of the electron current probability density in the case of photoionization of an atom in an external uniform static electric field. The resolution of PM is high enough for observing the quantum oscillatory spatial structure of the outgoing electron flux. We examine the Stark states of the magnesium atom and focus on PM features which are specific to the energy range just above the saddle-point energy. Particularly, the existence of $m$-dependent saddle-point thresholds is clearly demonstrated by following the evolution of the recorded angular distributions with energy. Furthermore, the outer inflection point radii of the radial distributions of the images are generally found to increase with energy monotonically but occasionally abruptly, signaling parabolic channel openings (above which these channels are converted to continua). This observation is in accord with recently reported quantum mechanical PM calculations for hydrogenic systems. More importantly, there are a number of nonmonotonic variations (local maxima) of the outer inflection point. The latter are attributed to the local emergence of an intensity halo at the outer part of the images, a feature relevant to the excitation of quasibound Stark states (resonances). The emergence of the halo for a given resonance is found to depend on the static electric field strength. These observations along with the difficulties in observing resonant effects on the recorded images of nonhydrogenic atoms, such as the medium-size magnesium atom, are discussed in detail.
\end{abstract}

DOI: 10.1103/PhysRevA.99.023428

\section{INTRODUCTION}

Photoionization microscopy (PM) is an experimental method designed to reveal the quantum structure of the twodimensional flux of very slow (meV) electrons ejected during the photoionization of a neutral atom in the presence of a uniform static electric field. The electron flux is recorded by a position-sensitive detector and under certain conditions the obtained image exhibits quantum interference effects which directly reflect the squared modulus of the electronic wave function. The term "microscopy" is justified because, along the direction of the static field, the Stark wave function of the photoionized electron extends over asymptotically large distances, while it is bound in the transverse direction. Moreover, just above the field-induced ionization threshold, continuum and quasibound Stark states (resonances) coexist and, therefore, PM may provide access to the wave function of one or the other. Quasibound states are, of course, of specific interest because they embody intrinsic properties of the atomic system under study.

The concept of PM was introduced during the early 1980s [1,2] and later analyzed in detail by Kondratovitch and Ostrovsky within the framework of the hydrogenic Stark effect, which is described in terms of so-called parabolic wave functions [3,4]. For hydrogen atom, images attributed unambiguously to Stark resonances were experimentally recorded fairly recently [5]. The distinct resonant character on these images is manifested via a noticeable increase of their radial extension, as well as the appearance of additional wave-

*Corresponding author: scohen@uoi.gr function nodes, while both of these characteristics are absent in the off-resonance, neighboring, continuum images below and just above a given resonance.

The observation of resonant images proved to be questionable for nonhydrogenic atoms because their Stark states are expressed as a mixture of quasibound as well as continuum hydrogenic parabolic states. The mixing between continua and resonances is caused by short-range interactions induced by the penetration of the excited electron's wave function into the ionic core. Consequently, any initially prepared quasidiscrete-state population autoionizes to the degenerate continua and the resonant features of the image may be obscured. The aforementioned mixing gets stronger with increasing core size and this partly explains why resonant effects were not observed in the PM images recorded with the heavy (atomic number $Z=54$ ) Xe atom [6], while being apparent in the one devoted to the light $(Z=3) \mathrm{Li}$ atom $[7,8]$. Nevertheless, theoretical calculations suggested that under certain conditions, it is possible to observe resonant effects in the alkali metals [9-11] or even Xe [12]. Particularly, they predicted that resonant manifestations are occasionally extremely sensitive to the field strength, as verified in the $\mathrm{He}$ $(Z=2)$ PM experiment [13] where specific resonant characters were recorded only near avoided crossings between pairs of resonances [14], one of them being effectively decoupled from the continua.

Evidently, the fact that resonant images were recorded so far solely in small complex atoms poses some limits on the usefulness of PM. In order to gradually overcome the difficulties met in heavier atoms, new experiments are certainly required that will profit from the knowledge acquired so far. For example, it is by now well established [7,8] that the resonant 
character of the recorded images is more readily visible in the energy range just above the ionization threshold, where the number of continua remains low. In fact, in order to avoid any misinterpretations, the characteristics of the continuum images need to be additionally studied within this energy range and in more detail than before. Motivated by the above reasoning, we present in this article experimental data concerning Stark resonances of the medium-size $\mathrm{Mg}$ atom $(Z=12)$. Particularly, by carefully examining the evolution of the radial extension of the images as a function of energy, we will demonstrate the strong connection between this extension and the emergence of additional continua, as well as with the presence of Stark resonances. Furthermore, as a byproduct, our two-photon excitation scheme out of the ground state of magnesium allowed for the population of Stark states with magnetic quantum numbers $m=0$ and $|m|=2$ and provided the opportunity to observe the dependence of the ionization threshold upon $m$. This dependence is already present in the classical expression of the saddle-point energy, although rarely used. Here this dependence is unambiguously proved by following the energy evolution of the angular distributions of the images.

The rest of the paper is organized as follows: In the next section, we provide a brief general description of photoionization microscopy and the Stark effect in the continuum by making use of a recent detailed presentation given in [15]. Section III describes schematically our experimental setup and procedure. Experimental results are presented and analyzed in Sec. IV. Finally, in Sec. V, our final remarks and discussion of possible directions for further work are given.

\section{THEORETICAL DESCRIPTION OF PHOTOIONIZATION MICROSCOPY}

Before going into the details of our experiment and observations, it is instructive to expose the principles of PM through a brief review of the quantum mechanics of the hydrogenic Stark effect. The Schrödinger equation describing a hydrogen atom in the presence of a homogeneous and static electric field $\mathbf{F}=F \mathbf{z}$ is written as (in a.u., $\hbar=e=m_{e}=1$ )

$$
\left[-\frac{1}{2} \nabla^{2}-\frac{Z}{r}+F z-E\right] \psi(\mathbf{r})=0,
$$

with $E$ the energy, $Z$ the nuclear charge $(Z=1$ for hydrogen), and $r=\left[x^{2}+y^{2}+z^{2}\right]^{1 / 2}$. This Schrödinger equation is separable in semiparabolic coordinates $\chi=[r+z]^{1 / 2}, v=$ $[r-z]^{1 / 2}$, and $\varphi=\tan ^{-1}(y / x)$, which are more advantageous for numerical calculations [15]. In these coordinates, the wave function may be written in the form

$$
\psi(\mathbf{r})=[2 \pi \chi v]^{-1 / 2} X(\chi) Y(v) e^{i m \varphi},
$$

with $m=0, \pm 1, \pm 2, \ldots$ the azimuthal quantum number, which remains a good quantum number ( $\psi$ of different $|m|$ are orthogonal to each other). Combining Eqs. (1) and (2), we obtain the following decoupled one-dimensional Schrödinger equations:

$$
\begin{aligned}
& {\left[-\frac{1}{2} \frac{d^{2}}{d \chi^{2}}+U_{X, \mathrm{eff}}(\chi)-2 Z_{1}\right] X(\chi)=0,} \\
& U_{X, \mathrm{eff}}(\chi)=\frac{4 m^{2}-1}{8 \chi^{2}}+\frac{F \chi^{4}}{2}-E \chi^{2},
\end{aligned}
$$

$$
\begin{aligned}
& {\left[-\frac{1}{2} \frac{d^{2}}{d v^{2}}+U_{Y, \mathrm{eff}}(v)-2 Z_{2}\right] Y(v)=0,} \\
& U_{Y, \mathrm{eff}}(v)=\frac{4 m^{2}-1}{8 v^{2}}-\frac{F v^{4}}{2}-E v^{2},
\end{aligned}
$$

where the separation constants $Z_{1}$ and $Z_{2}$ are related through $Z_{1}+Z_{2}=Z$. The electron may escape to infinity along the $v$ coordinate. Therefore, the problem is a scattering one and it is solved for a predetermined energy $E$ at fixed values of $m$ and field strength $F$.

The $\chi \rightarrow \infty$ asymptotic shape of the effective potential $U_{X \text {,eff }}$ implies that the wave function $X(\chi)$ is bound. The solution of the differential Eq. (3a) involves the quantization of $Z_{1}$, thus also fixing $Z_{2}$. The obtained $Z_{1}^{n_{1},|m|}$ set is characterized by the number of nodes, $n_{1}=0,1,2 \ldots$, of the wave functions $X_{n_{1},|m|}$ in the interval $(0, \infty)$. Furthermore, for given $E, m$, and $F$, the shape of $U_{X \text {, eff }}$ causes the spatial extensions of $X_{n_{1},|m|}$ to grow with increasing $n_{1}$.

Features specific to the resonant nature of the excited level in the image are more easily visible just above the classical saddle-point energy $[5,7,8] E_{s p}^{c l}=-2[Z F]^{1 / 2}$ a.u., in a region where the number of semiparabolic $n_{1}$ channels contributing to the image remains low. At this negative energy range, the effective potential $U_{Y \text {,eff }}$ exhibits an inner well and a barrier (see Fig. 1 of [15]). As a consequence, each $n_{1}$ channel is associated to a threshold $E_{\mathrm{thr}}^{n_{1},|m|}$, found by solving the equation $E=-2\left[Z_{2}^{n_{1},|m|}(F, E) F\right]^{1 / 2}[15]$. For $E>E_{\mathrm{thr}}^{n_{1},|m|}$, the electron escapes over the barrier and its continuum state is characterized only by the pair $\left(n_{1}, m\right)$. At the opposite, for $E<E_{\mathrm{thr}}^{n_{1},|m|}$ the electron may escape solely via tunneling through the barrier. In this case, there are specific values of $Z_{2}^{n_{1},|m|}$ which match the eigenvalues of the inner well of $U_{Y \text {,eff }}$. Then, the probability of finding the electron within the inner well increases dramatically, the electron is trapped at short distances, and its quasibound state (resonance) exhibits an appreciable lifetime. These states are then characterized by an enlarged set of three quantum numbers $\left(n_{1}, n_{2}, m\right)$, where $n_{2}$ is the number of nodes of $Y(v)$ inside the inner well. Within $E_{s p}^{c l} \leqslant E \leqslant 0$, these quasidiscrete states coexist with continuum ones corresponding to different $n_{1}$.

Photoionization microscopy relies on the recording of the photocurrent density,

$$
\begin{aligned}
j_{v_{\mathrm{det}}}(\varphi, \chi) & \equiv j\left(\varphi, v=v_{\mathrm{det}}, \chi\right) \\
& \left.\propto i\left[\psi \frac{\partial \psi^{*}}{\partial v}-\psi^{*} \frac{\partial \psi}{\partial v}\right]\right|_{v=v_{\mathrm{det}}},
\end{aligned}
$$

at a macroscopic distance, along a given constant $v=v_{\text {det }} \rightarrow$ $\infty$ paraboloid. The latter crosses the $z$ axis at $z=z_{\text {det }}=$ $-v_{\text {det }}^{2} / 2$, where the detector is located [15]. It turns out that as $v_{\text {det }} \rightarrow \infty, j_{v_{\text {det }}}$ may be written as

$$
j_{v_{\mathrm{det}}}(\varphi, \rho) \propto \frac{1}{\chi}\left|\sum_{m} \sum_{n_{1}=0}^{\infty} c_{n_{1}}^{m}(\varphi) X_{n_{1},|m|}(\chi)\right|^{2},
$$

where $\rho=\chi v_{\text {det }}$ denotes the radius on the detector. The complex weights $c_{n_{1}}^{m}$ are proportional to the matrix elements describing the photoexcitation process from a particular initial 


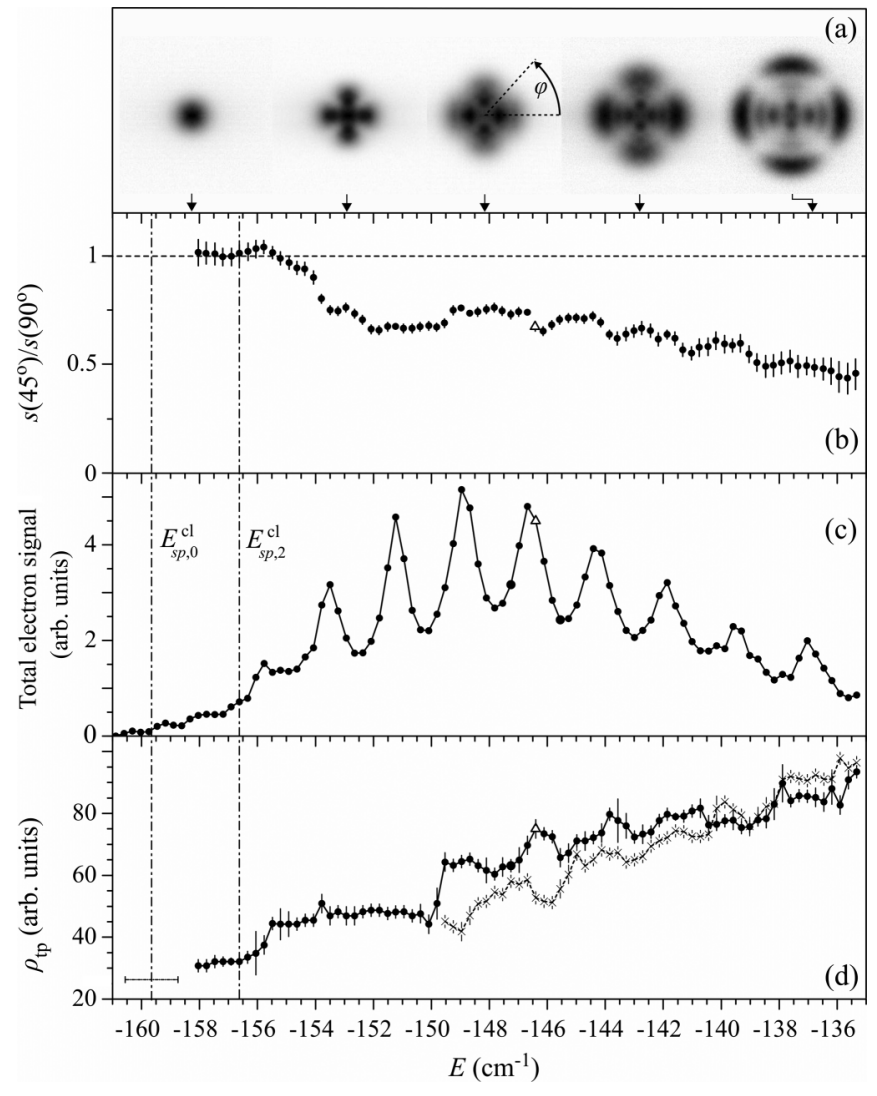

FIG. 1. (a) Near-threshold symmetrized images recorded with $\sigma$ polarization $(|m|=0,2$ final Stark states) at selected energies, marked with arrows. Symmetrization is applied for signal-to-noise ratio improvement and refers to the average between the four quadrants of each image; the latter expected to be vertically and horizontally symmetric. The gray scale is stretched from zero (white) to $100 \%$ (black) for each image. (b) Ratio $s\left(45^{\circ}\right) / s\left(90^{\circ}\right)$ [the function $s(\varphi)$ is defined in Eq. (8)], expected to be equal to unity for purely $m=0$ images and equal to zero for purely $|m|=2$ images (see text). (c) Near-threshold total electron signal proportional to $\sigma_{t o t}(\sigma$ polarization). Each point corresponds to a recorded image. (d) Outer inflection points of radial distributions $[P(\rho)]^{1 / 2}=[\rho R(\rho)]^{1 / 2}$, for $\sigma$ polarization (black circles, solid line) and $\pi$ polarization (crosses, dashed line). In (b)-(d), the $m=0\left(E_{s p, 0}^{c l}=E_{s p}^{c l}\right)$ and $|m|=2\left(E_{s p, 2}^{c l}\right)$ saddle-point energies (estimated as described in the text) are indicated by vertical dash-dotted lines and the open triangle denotes the energy location of a quasibound state whose image and radial distribution $R(\rho)$ are given in Fig. 2 (see text).

state to the final Stark states. The density $j_{v_{\text {det }}}$ (i.e., the image recorded on the detector) incorporates both angular and radial information and has the form of an interferogram. Although the coherent summation over $m$ in Eq. (5) implies that $m$ interferences are also possible (depending on the accessible values of $m$ [12]), PM is primarily concerned with the interference pattern along the radius $\rho$. This pattern can be more easily studied via the radial distribution

$$
R(\rho)=\int_{0}^{2 \pi} j_{v_{\mathrm{det}}}(\varphi, \rho) d \varphi
$$

which can be regarded as the electron's momentum distribution transversely to the electric field $[15,16]$. In fact, the term "radial distribution" is more frequently employed for $P(\rho)=$ $\rho R(\rho)$, which is proportional to the number of electrons hitting the detector within the $[\rho, \rho+d \rho]$ interval and can also be employed for probing radial interferences. Additionally, resonances and continua also manifest themselves in the total ionization cross section that can be obtained by integrating $j_{v_{\text {det }}}$ over the whole surface of the $v_{\text {det }}$ paraboloid, i.e.,

$$
\sigma_{\text {tot }}=\int j_{v_{\mathrm{det}}}(\varphi, \rho) d S,
$$

where $d S$ denotes the surface element of this paraboloid [15]. Note that the aforementioned $m$-interference effects vanish in Eqs. (6) and (7) due to the $m$ - and $n_{1}$-orthonormality properties of the Stark wave functions $\psi(\mathbf{r})$.

In practice the $n_{1}$ sum in Eq. (5) is finite. Consider, for example, the case where at a given energy $E$ there is no quasibound state, but only continua with quantum numbers $0 \leqslant n_{1} \leqslant n_{1}^{\mathrm{o}}$ (i.e., $E_{\mathrm{thr}}^{n_{1}^{\mathrm{o}}} \leqslant E \leqslant E_{\mathrm{thr}}^{n_{1}^{\mathrm{o}}+1}$ ). Then, evidently the sum runs only up to $n_{1}^{o}$ and several out of the available $X_{n_{1},|m|}$ waves contribute to $j_{v_{\text {det }}}$ with comparable weights. Nevertheless, $X_{n_{1}^{\circ},|m|}$ dominates the interferogram (particularly at large values of $\rho$ ) which exhibits $n_{1}^{\circ}$ dark fringes [8]. For fixed $F, n_{1}^{\mathrm{o}}$ and its associated outer-image radius increase monotonically with energy [6,8]. Finally, recent theoretical studies [15] have shown that just above $E_{s p}^{c l}$, the nonresonant outer-image radius may occasionally increase discontinuously in the neighborhood of the thresholds $E_{\mathrm{thr}}^{n_{1},|m|}$. This will be clearly illustrated in the present article.

Assume now that apart from the $0 \leqslant n_{1} \leqslant n_{1}^{\text {o }}$ continua, a single resonance is additionally present at $E$. The resonance is characterized by the set $\left(n_{1}^{\text {res }}, n_{2}^{\text {res }}, m_{\text {res }}\right)$ where, since $E<$ $E_{\mathrm{thr}}^{n_{1}^{\text {res }}}$ and $E_{\mathrm{thr}}^{n_{1}^{\mathrm{o}}} \leqslant E$, it necessarily holds that $n_{1}^{\text {res }}>n_{1}^{\mathrm{o}}$. Note that $n_{2}^{\text {res }}$ cannot be extracted from the measurement of $j_{v_{\mathrm{det}}}$. As a general rule, for the hydrogen atom, $\left|c_{n_{1}^{\text {res }}}^{m_{\text {res }}}\right|$ is several orders of magnitude larger than any of the $\left|c_{n_{1}}^{m}\right|$ 's for $0 \leqslant n_{1} \leqslant$ $n_{1}^{\mathrm{o}}$. Hence, the resonance largely dominates the background formed by these continua and this is also evident in $\sigma_{\text {tot }}$. More importantly, a strong modification of the interference pattern is expected as the energy is scanned across the resonance. Indeed, just below and just above the resonance, $j_{v_{\text {det }}}$ will exhibit $n_{1}^{\mathrm{o}}$ dark fringes. On-resonance, however, it will exhibit mostly the characteristics of $X_{n_{1}^{\text {res }},\left|m_{\text {res }}\right|}$ and their number will be equal to $n_{1}^{\text {res }}$. Additionally, because $n_{1}^{\text {res }}>n_{1}^{\mathrm{o}}$, the radial extension of $X_{n_{1}^{\text {res }},\left|m_{\text {res }}\right|}$ is larger than any of the other $X_{n_{1},|m|}$ functions and we expect a noticeable on-resonance enhancement of the image size.

Let us finally focus our attention to nonhydrogenic atoms for which the forms of Eqs. (5)-(7) remain apparently unchanged. Nevertheless, the aforementioned mixing between quasibound states and continua results in a drastic decrease of the magnitude of $c_{n_{1}^{\text {res }}}^{m_{\text {res }}}$, which may become comparable to the magnitude of the weights of the continua. Consequently, despite the appearance of the resonance in $\sigma_{\text {tot }}$, the characteristics of $X_{n_{1}^{\text {res }},\left|m_{\text {res }}\right|}$ may not dominate the on-resonance image. In the $\mathrm{Li}$ experiment [7,8] these characteristics were weak but observable. Moreover, the modifications of $j_{v_{\text {det }}}(\varphi, \rho)$ and $R(\rho)$ or $P(\rho)$ caused by the presence of the resonance are expected to be fainter and fainter as the mixing gets stronger. This might explain the absence of resonant effects 
in Xe images [6]. On the other hand, the successful recording of resonant images in the helium atom was based on the decoupling of the resonance from the degenerate continua in the neighborhood of avoided crossings [13]. Additionally, for the alkali metals, it was suggested that for a given $\left(n_{1}, n_{2}, m\right)$ resonance, there exist ranges in the $\{E, F\}$ space where the coupling is apparently reduced and the observation of images exhibiting specific resonant characteristics is more probable [10]. Let us finally note that in order to get closer to the hydrogenic case and to enhance specific resonance features, it is advisable, when possible, to configure the experiments in a manner where high- $|m|$ states are populated, suppressing contributions from highly core-penetrating low- $\ell$ orbitals. Below we describe the application of some of the above ideas to our Mg PM experiment.

\section{EXPERIMENTAL SETUP AND PROCEDURE}

The experimental setup is similar to the one described in Refs. $[8,15,17]$. Magnesium vapor is produced in an electrically heated stainless-steel oven mounted at the top of the laser-atom interaction region. The background pressure in this region is $\approx 7 \times 10^{-7}$ mbar. The produced thermal beam of $\mathrm{Mg}$ atoms interacts with the frequency-doubled radiation delivered by a pulsed Nd:yttrium aluminum garnet (YAG)pumped dye laser operating at a repetition rate of $10 \mathrm{~Hz}$. The fundamental visible $(610-670 \mathrm{~nm})$ radiation pulses have $\sim 5 \mathrm{~ns}$ duration and a linewidth of $\sim 0.2 \mathrm{~cm}^{-1}$. A part of it is guided towards a wavelength calibration system consisting of a Fabry-Perot interferometer providing relative calibration (free spectral range $0.4729(2) \mathrm{cm}^{-1}$ ) and a discharge lamp offering absolute calibration via the one-photon optogalvanic spectrum of Ne. The frequency-doubled UV radiation $(305-335 \mathrm{~nm})$ is produced by a potassium dihydrogen phosphate (KDP) crystal and its maximum pulse energy amounts to $\sim 1 \mathrm{~mJ}$. Its separation from the visible one as well as its pulse energy regulation is achieved by means of appropriate filters. Next, the UV beam passes through an alpha-betabarium-borate (alpha-BBO) Rochon prism linear polarizer and a double-Fresnel rhomb (acting as a $\lambda / 2$ retarder) for purifying and rotating its linear polarization, respectively. Subsequently, it is focused to the vacuum chamber via an in-vacuum $\sim 5 \mathrm{~cm}$ focal length lens and excites the final $3 s E k$ Stark states of $\mathrm{Mg}$ atom (with $E k$ denoting the $\mid k>$ Rydberg electron Stark state excited at energy $E$ ) via a nonresonant two-photon scheme out of its $3 s^{2}{ }^{1} S_{0}$ ground state. The combination of frequency-doubling and two-photon excitation leads to a spectral width of about $0.8 \mathrm{~cm}^{-1}$.

The atomic and laser beams are both perpendicular to the axis of the electron spectrometer. The latter is based on a standard three-electrode velocity-map imaging (VMI) spectrometer design [18]. Photoionization takes place in the center between a solid repeller plate and an extractor plate with a hole in its center. These electrodes are biased at appropriate voltages [17] and they are followed by a grounded third electrode identical to the extractor plate. The static field in the interaction region is inhomogeneous. This is necessary for the spectrometer to meet the VMI focusing conditions $[17,18]$. Nevertheless, in the vicinity of the limited laser-atom interaction volume, the field may be considered as being nearly constant, but its actual value is rather difficult to estimate accurately based on the applied voltage values. Its more accurate determination is done via experimental measurements (see Sec. IV). Photoionized electrons are accelerated by the field towards the end of a field-free drift tube. An electrostatic einzel lens is placed about halfway through the tube for magnifying the recorded images. At the end of the tube, the electrons are detected by a twodimensional position-sensitive detector made of a tandem microchannel plate assembly and a phosphor screen. A CCD camera records the two-dimensional (2D) distribution of light spots on this screen. Recorded images are transferred to a computer, where they are accumulated over several-thousand laser shots. The entire spectrometer is shielded by a double $\mu$-metal layer, resulting in a residual magnetic field $<1 \mu \mathrm{T}$ in its interior.

\section{RESULTS AND DISCUSSION}

As mentioned earlier [8], the energy range just above $E_{s p}^{c l}$ is the most advantageous one for recording images exhibiting resonant effects. The efforts of the present study are, therefore, focused on this range which is characterized by a small number of continua and accompanying fringes. Figure 1 summarizes our measurements. Figure 1(a) shows nonresonant experimental images displaying the well-known monotonous increase of the number of radial nodes (destructive interference or "dark" fringes) with the energy. These images were recorded with the linear laser polarization perpendicular to the field axis (along the vertical axis in the figure). For this $\sigma$-polarization configuration, the dipole excitation selection rule is $\Delta m= \pm 1$ per photon, and leads to the excitation of $|m|=0$ and 2 final Stark states after two-photon absorption. Noticeably, the lowest-energy images are disc shaped and show no angular dependence, as it would be expected for $m=0$ states $[4,5,8,15]$. However, for energies higher than $\approx-155.5 \mathrm{~cm}^{-1}$, all images exhibit a $\cos ^{2}(2 \varphi)$, crosslike, angular distribution that is distinctive of the $|m|=2$ character $[12,15]$. The energy where the transition occurs can be phenomenologically quantified by defining the angular function,

$$
s(\varphi) \equiv \int_{0}^{\infty} j_{v_{\mathrm{det}}}(\varphi, \rho) d \rho,
$$

and by employing the ratio $s\left(45^{\circ}\right) / s\left(90^{\circ}\right)$. The latter is expected to be equal to unity for purely $m=0$ images and equal to zero for purely $|m|=2$ images exhibiting a node at $\varphi=45^{\circ}$. Figure 1 (b) shows this ratio as a function of the energy and it is easy to note its deviation from unity and its rapid decrease for $E>-155.5 \mathrm{~cm}^{-1}$. This observation is consistent with the $|m|$-dependent classical saddle-pointenergy formula [19],

$$
E_{s p,|m|}^{c l} \approx E_{s p}^{c l}\left[1-\frac{|m|}{2} F^{1 / 4}-\frac{3}{32} m^{2} F^{1 / 2}\right],
$$

predicting that $E_{s p, 0}^{c l}=E_{s p}^{c l}<E_{s p, 2}^{c l}$. The energy of the first image where a quantifiable signal could be obtained supplied a first estimate of the threshold $E_{s p}^{c l}$ and its uncertainty. This provided a value for the field strength $F$ and subsequently for $E_{s p, 2}^{c l}$ through Eq. (9). Both $m=0$ and $|m|=2$ thresholds 
are indicated by vertical dash-dotted lines in Fig. 1. The estimated value of $E_{s p, 2}^{c l}$ agrees with the one predicted via the $s\left(45^{\circ}\right) / s\left(90^{\circ}\right)$ ratio within $\approx 1 \mathrm{~cm}^{-1}$ and both procedures converge to a field value $F=680 \pm 10 \mathrm{~V} / \mathrm{cm}$.

The above discussion implies that for $E_{s p}^{c l} \leqslant E<E_{s p, 2}^{c l}$, one expects the sole presence of $m=0$ continua (and possibly resonances), while for $E \geqslant E_{s p, 2}^{c l}$, we expect the presence of both $|m|=0$ and 2 Stark states. In fact, although Eq. (9) is long-ago known, and $m$-dependent field-ionization thresholds of individual Rydberg states were demonstrated in the past $[19,20]$, to the best of our knowledge this equation has not been tested and validated on the basis of angular distributions. Indeed, in the present work, the existence of $m$-dependent saddle-point thresholds is proved by the fairly brutal change in angular distribution around $-155.5 \mathrm{~cm}^{-1}$, in accord with the predictions of Eq. (9). Furthermore, the simultaneous excitation of $|m|=0$ and 2 states may lead to an $m$-beating effect (see Eq. (5) and [12]). This can be observed in the crosslike images of Fig. 1(a), where the fringe contrast along their horizontal direction appears to be somewhat higher than the contrast along their vertical direction (as it was already observed in [15]; see Fig. 4 therein). However, apart from this weak beating effect, the recorded images show that the $|m|=2$ character dominates over the $m=0$ one.

Images were acquired with an energy step of $\approx 0.3 \mathrm{~cm}^{-1}$. For each image, the total electron signal is obtained by (angularly and radially) integrating over the whole detector surface. The pulse energy was reduced in order to avoid saturation effects. Under such conditions, the integrated signal is proportional to the total ionization cross section $\sigma_{\text {tot }}(E)$ [Eq. (7)] and it is given in Fig. 1(c). It has been verified that this spectrum is identical to the one of $\mathrm{Mg}^{+}$ions, obtained by reversing the polarity of the voltages applied to the spectrometer and otherwise identical conditions. The recorded cross section consists of a series of almost equidistant broad spectral lines superimposed on a background of slowly varying magnitude. The apparent resonant-to-background ratio is $\sim 2: 1$. It is noteworthy that a number of minima of the ratio $s\left(45^{\circ}\right) / s\left(90^{\circ}\right)$ in Fig. 1(b), which characterize $|m|=2$ resonances, lie very close to the resonant maxima of the spectrum of Fig. 1(c). Typical linewidths of $\approx 1 \mathrm{~cm}^{-1}$ or more are observed, slightly exceeding the anticipated spectral resolution $\left(\approx 0.8 \mathrm{~cm}^{-1}\right)$ and indicating the blend of several resonances within a given line and/or a small inhomogeneous broadening due to the inhomogeneity of the electric field. The corresponding spectrum with the linear laser polarization parallel to the field axis (not shown) exhibits resonances at almost the same locations, but with occasionally different line profiles. Its apparent resonant-to-background ratio is slightly lower than that of the $\sigma$-polarization spectrum of Fig. 1(c). This last $\pi$-polarization configuration (dipole selection rule $\Delta m=0$ ) leads to the excitation of $m=0$ final Stark states only and the spectrum presents some similarities with the one obtained via single-photon excitation and much higher field strengths [21]. The recorded images show no angular dependence. It may be concluded that the resonant energies of $|m|=2$ and $m=0$ quasibound states lie very close to each other, i.e., they are nearly degenerate. Such a quasidegeneracy of resonances has already been predicted by earlier semiclassical theoretical work and it is anticipated to occur for either hydrogenic or multielectron atom near-threshold Stark spectra [22-24]. The same works also showed that this quasidegeneracy is to be expected for the $E_{\mathrm{thr}}^{n_{1},|m|=2}$ and $E_{\mathrm{thr}}^{n_{1}+1, m=0}$ channel thresholds as well.

The above information is useful when examining the radial extension of the images which is quantified here in a fashion similar to that of Ref. [8]. Specifically, this radial extension is presently defined as the radius of the outermost inflection point of the function $[P(\rho)]^{1 / 2}=[\rho R(\rho)]^{1 / 2}$ (i.e., the outermost radius for which $\left.d^{2}\left\{[P(\rho)]^{1 / 2}\right\} / d \rho^{2}=0\right)$. Our present choice is guided by the fact that for $\rho \rightarrow \infty$ (and, consequently, $\chi \rightarrow \infty)[P(\rho)]^{1 / 2} \propto\left|X_{n_{1}^{\max },|m|}\right|$, where, for a given $|m|, n_{1}^{\max }$ denotes the larger $n_{1}$ value in the sum of Eq. (5) for which the corresponding coefficient $c_{n_{1}}^{m}$ is nonnegligible. Therefore, the outermost inflection point of the function $[P(\rho)]^{1 / 2}$ should correspond to the outer turning point $\rho_{\mathrm{tp}}$ of $X_{n_{1}^{\max },|m|}$. In Ref. [8], the outermost inflection point of $P(\rho)$ itself was used instead, and this inflection point does not correspond to $\rho_{\text {tp }}$, although it may be related to it. Note that in any choice, inflection points are independent of the magnitude of $P(\rho)$. In the absence of a resonance, we expect that $n_{1}^{\max }=n_{1}^{\mathrm{o}}$ and the energy evolution of $\rho_{\mathrm{tp}}$ may probe $n_{1}$-channel openings. On the other hand, in the presence of a resonance with a non-negligible coefficient $c_{n_{1}^{\text {res }}}^{m_{\text {res }}}$, it is expected that $n_{1}^{\max }=n_{1}^{\text {res }}>n_{1}^{\mathrm{o}}$ and $\rho_{\mathrm{tp}}$ will be affected by the spatial extension of the $X_{n_{1}^{\text {res }},\left|m_{\text {res }}\right|}$ wave.

The $\rho_{\text {tp }}(E)$ curves obtained from the presently measured images are given in Fig. 1(d). We are interested mainly in the $\sigma$-polarization data that are dominated by the $|m|=2$ Stark states for which $n_{1}$-channel mixing due to the penetration of the excited electron to the ionic core is expected to be much weaker than for the $m=0$ states. It may be, therefore, anticipated that the magnitude of $\left|c_{n_{1}^{\text {res }}}^{m_{\text {res }}=2}\right|$ would be sufficiently higher than the magnitude of the nonresonant weights $\left|c_{n_{1}}^{m=2}\right|$ in order for the resonant signatures to be observable, even under the presence of a "parasitic" $m=0$ nonresonant contribution. The overall trend of these $\sigma$-polarization data reveals a generally monotonous (within error bars) but occasionally discontinuous and steplike energy evolution of $\rho_{\text {tp }}$ (see particularly the steps at $\approx-155$ and $\approx-149 \mathrm{~cm}^{-1}$ ). This behavior seems to persist with increasing energy, but with a reduced contrast which makes it difficult to analyze and comment. It is the small energy step employed here that allowed this observation of these discontinuities. As mentioned above, the occasional occurrence of these $\rho_{\text {tp }}$ jumps was predicted quite recently in theoretical calculations devoted to the hydrogenic Stark effect [15]. It was shown that they correspond to $n_{1}$ channel openings which occur in the neighborhood of each $E_{\mathrm{thr}}^{n_{1},|m|}$ threshold. For example, the abrupt increase of $\rho_{\mathrm{tp}}$ at $\approx-155 \mathrm{~cm}^{-1}$ corresponds to the opening of the $|m|=2$, $n_{1}=0$ channel (and also of the nearby $|m|=0, n_{1}=1$ one). The same calculations, however, have also shown that not all of the jumps are always observable. For the present data, such is the case for the $|m|=2, n_{1}=1$ channel opening that, according to the hydrogenic trends, would be expected at $\approx-153 \mathrm{~cm}^{-1}$. Apparently, the initial state and excitation pathway determine to a large extent which of the jumps would exhibit a non-negligible magnitude [15]. 
(b)

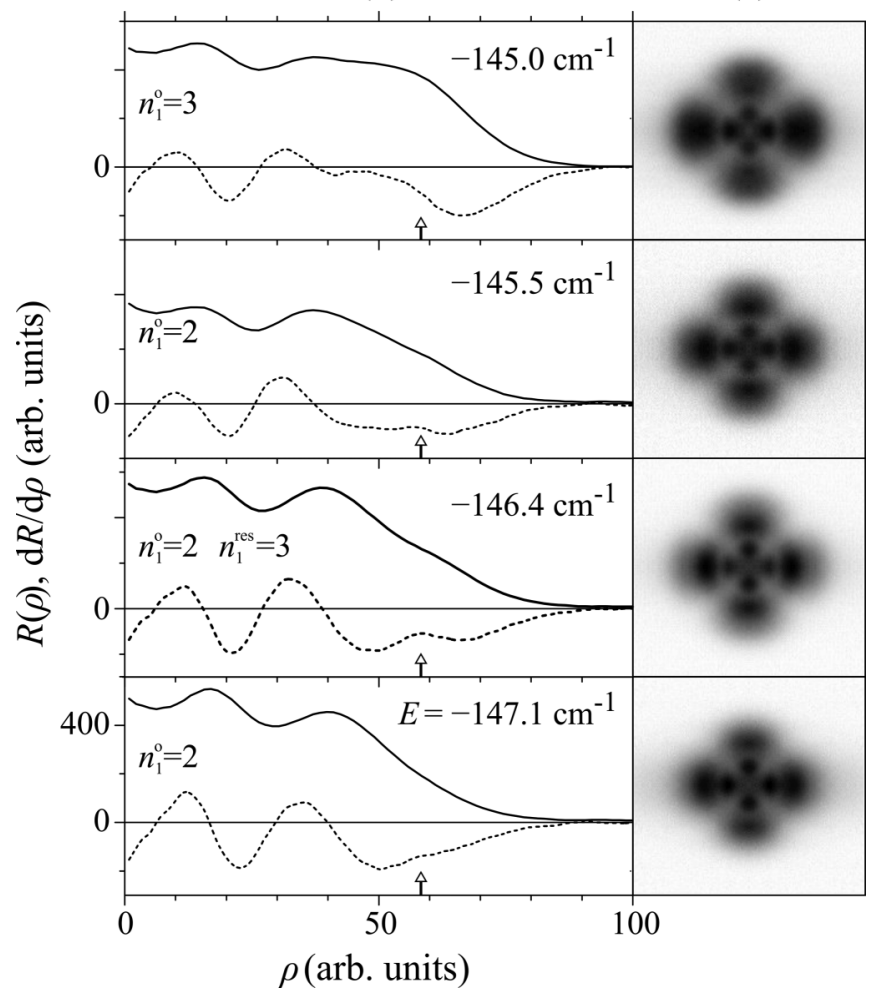

FIG. 2. (a) Symmetrized images and (b) corresponding radial distributions $R(\rho)$ defined in Eq. (6), recorded with $\sigma$ polarization in the vicinity of the quasibound state at $-146.4 \mathrm{~cm}^{-1}$ [marked with an open triangle in Figs. 1(b)-1(d)]. All $y$-axis scales are the same and the relative magnitudes of $R(\rho)$ are meaningful. For each image in (a), however, the gray scale is stretched from zero (white) to $100 \%$ (black). In (b), the derivatives $d R / d \rho$ are also drawn with dashed lines and the relevant $n_{1}^{\mathrm{o}}$ and $n_{1}^{\text {res }}$ values are noted. Upward white-head arrows mark the $\rho \sim 57$ arbitrary length unit where the derivative of the resonant radial distribution $\left(E=-146.4 \mathrm{~cm}^{-1}\right)$ shows an additional extremum. This extremum indicates the presence of a weak outer "bump" which is related to the external halo of the corresponding resonant image. Note that the $n_{1}=3$ channel opening $\left(E=-145.0 \mathrm{~cm}^{-1}\right)$ is characterized by the onset of appearance of an additional fringe at $\rho<50$ arbitrary length units.

Even more interesting than the monotonous but steplike increase of $\rho_{\text {tp }}$ are the few cases where the turning point radius evolves nonmonotonously and peaks near the maxima of the spectral lines of $\sigma_{\mathrm{tot}}$. As it emerges from earlier theoretical predictions $[4,10,12,25]$ and our presentation in the theoretical section above, this kind of behavior characterizes the excitation of quasibound resonances belonging to closed $n_{1}$ channels and ionizing mostly by tunneling. This feature has been clearly demonstrated for the hydrogen atom, both experimentally [5] and theoretically (see, for example, Fig. 2 of [15]), and it appears indeed to be more specific of a resonant property. In most such cases of Fig. 1(d), the turning point modulation amplitude is rather small as compared with the estimated error bars. The fairly clear $\rho_{\text {tp }}$ maximum around $-146.4 \mathrm{~cm}^{-1}$, lying between the estimated locations of the $n_{1}=2$ and $n_{1}=3,(|m|=2)$ channel openings, represents a noticeable exception. This maximum occurs near the peak and slightly on the blue side of the corresponding resonance of $\sigma_{\text {tot }}$ and it is absent in the $\rho_{\mathrm{tp}}(E)$ curve recorded with $\pi$ polarization [see Fig. 1(d)]. In fact, this $\pi$-polarization $\rho_{\mathrm{tp}}(E)$ curve exhibits two dips and a weak local maximum around $-147 \mathrm{~cm}^{-1}$. The origin of this structure is not clear as yet. It could stem from $n_{1}$-channel openings and/or the presence of an $m=0$ resonance located at $\approx-147 \mathrm{~cm}^{-1}$, but the inspection of the corresponding $m=0$ images is inconclusive. In any case, we may confidently conclude that the nonmonotonous behavior of the $\sigma$-polarization $\rho_{\mathrm{tp}}(E)$ curve at $\approx-146.4 \mathrm{~cm}^{-1}$ probes an $|m|=2$ resonance [this conclusion is also in accord with the $|m|=2$ resonant minimum observed in Fig. 1(b) at this energy]. Figure 2 depicts the evolution of selected images [Fig. 2(a)] and radial distributions $R(\rho)$ [Fig. 2(b)] in the vicinity of this resonance. The three lower radial distributions of the plot (below the resonance at $-147.1 \mathrm{~cm}^{-1}$, on resonance and above the resonance at $-145.5 \mathrm{~cm}^{-1}$ ) look much alike, apart from a small but important difference. Specifically, the on-resonance image exhibits a weak but observable intensity halo at its outer part, which translates into a weak extra outer "bump" in the distribution $R(\rho)$. This bump is responsible for the observed maximum of $\rho_{\mathrm{tp}}$ in Fig. 1(d). Nevertheless, the fact that it constitutes an additional bright fringe (implying a change of $n_{1}^{\max }=$ $\left.n_{1}^{\text {res }}=n_{1}^{\mathrm{o}}+1\right)$ is not so obvious. It becomes more apparent, however, by inspecting the derivatives $d R(\rho) / d \rho$ which are also plotted in Fig. 2(b) with dashed lines. The interesting feature of the derivatives is their extremum that originates from the extra node and occurs at $\rho \sim 57$ arb. length units (alu). This extremum appears over of the whole $-147 \leqslant E \leqslant$ $-145.5 \mathrm{~cm}^{-1}$ range, but it is clearly visible at $-146.4 \mathrm{~cm}^{-1}$, while hardly visible at the nonresonant derivatives. We may conclude that the resonant character (as quantified by the magnitude of $\left.\left|c_{n_{1}^{\text {res }}}^{m_{\text {res }}}\right|\right)$ is spread across the spectral line and peaks on the resonance. Such spreads of resonant character were also observed in calculations concerning resonant PM images of the Li atom [8]. At higher energy and just outside the resonant profile [Fig. 2(b), $E=-145.0 \mathrm{~cm}^{-1}$ ], the aforementioned local extremum of $d R(\rho) / d \rho$ at $\rho \sim 57$ alu disappears. Instead, we evidence an increased radial extension of $R(\rho)$ and the onset of appearance of an additional fringe at $\rho<50$ alu. This additional fringe signals the next $n_{1}$-channel opening which is indeed observed at even higher-energy images. Thus, it appears that the resonance at $-146.4 \mathrm{~cm}^{-1}$ (apparently with $n_{1}^{\text {res }}=3$ ) belongs to the last quasibound state before the opening of the corresponding channel.

Let us finally note that apart from the present study, the appearance of the intensity halo on the images was also used as the principal manifestation of resonant character in the PM study of lithium $[7,8]$. Here, we traced its importance by recording similar sets of images at several field strengths that are up to $\pm 5 \mathrm{~V} / \mathrm{cm}$ apart from the field employed for the measurements of Figs. 1 and 2. Interestingly, the halo of the presently examined resonance is found to wash out in these measurements. Such a behavior was theoretically predicted for multielectron atoms [10] and, rather intuitively, implies that the coupling between quasibound states and continua depends on and varies with $F$. 


\section{CONCLUSION}

We have presented a photoionization microscopy study of the Stark states of Mg. These states were two-photon excited from the atomic ground state and located just above the saddle-point energy. In fact, for linear laser polarization perpendicular to the direction of the static electric field, the evolution of the angular distributions of the recorded images with energy has confirmed the long-ago predicted [19] existence of $m$-dependent saddle-point energies (one for $m=$ 0 and one for $|m|=2$ in the present case). Moreover, the outer turning points of the radial distributions of the images were overall found to monotonically increase with energy. Occasionally, however, they increased discontinuously and each observed jump signalled the opening of an $n_{1}$ channel, the latter becoming a continuum one. This observation is in complete accord with recent theoretical calculations [15] predicting that just above the saddle-point energy, channel openings may frequently (but not always) lead to abrupt increases of the radial extensions of the images. Finally, there were a number of cases where the outer turning points showed a nonmonotonic variation near the resonant maxima of the total ionization cross section. This variation is due to an on-resonance appearance of an intensity halo at the outer part of the relevant images. The halo may be interpreted as an additional bright fringe, which is characteristic of the quasibound state participating non-negligibly to the photocurrent probability density. The resonant character is found to spread over the width of a given spectral line.

The present results concerning the medium-size $\mathrm{Mg}$ atom $(Z=12)$ and the resonant manifestations exhibited by the recorded photoionization microscopy images of the small Li atom $(Z=3)$ [7,8] are both found to be rather weak with respect to the corresponding manifestations in hydrogen atom [5]. Furthermore, resonant signatures on the recorded $\mathrm{Mg}$ images are apparently found to be somewhat weaker than those in Li. Leaving aside ionic-core size effects, this may be explained by the larger effective laser linewidth of the present study, as well as by the increased "parasitic" ionization signal emerging from the simultaneous excitation of the nonresonant $m=0$ contribution, in addition to the $|m|=$ 2 resonant and nonresonant contributions. On the contrary, excitation of single- $|m|$ Stark states was possible in the $\mathrm{Li}$ experiment $[7,8]$. These studies lead to the conclusion that in order to unbury the resonant character from the recorded images, a number of precautions have to be taken and a number of special excitation strategies have to be devised. For example, it appears that the spectrally sharp Stark resonances require a spectral laser resolution much better than $0.1 \mathrm{~cm}^{-1}$. Ideally, (possibly pulsed amplified) cw lasers could be employed in conjunction with single-photon transitions, or, when necessary, multistep, multicolor, excitation schemes. In terms of the appropriate field strengths, the present study followed the earlier theoretical suggestions [10] and found indeed that there are values of $F$ for which the resonant manifestations may be somewhat optimized. Nevertheless, the effect was much weaker than predicted. Clearly, working near avoided crossings, as in the He experiment [13], presents a quite promising direction, which appears to be potentially applicable to any nonhydrogenic atom. Finally, another interesting possibility is to achieve excitation near avoided crossings by means of bichromatic laser fields and apply phase-sensitive coherent control techniques. By employing such schemes, the continuum excitation could be minimized, in favor of the excitation of the quasibound states [26]. We are currently working towards these directions.

\section{ACKNOWLEDGMENTS}

The experiment was performed at the Central Laser Facility of the University of Ioannina. The authors would like to thank F. Lépine for his valuable suggestions and comments and K. Ferentinou for her help in the data analysis. Technical assistance from P. Triantafillou and E. Dimitriadis is also gratefully acknowledged.
[1] I. I. Fabrikant, Z. Eksp. Teor. Fiz. 79, 2070 (1980) [Sov. Phys. JETP 52, 1045 (1980)].

[2] Yu. N. Demkov, V. D. Kondratovich, and V. N. Ostrovsky, Pis'ma Z. Eksp. Teor. Fiz. 34, 425 (1981) [JETP Lett. 34, 403 (1981)].

[3] V. D. Kondratovich and V. N. Ostrovsky, J. Phys. B 17, 1981 (1984); 17, 2011 (1984); 23, 21 (1990).

[4] V. D. Kondratovich and V. N. Ostrovsky, J. Phys. B 23, 3785 (1990).

[5] A. S. Stodolna, A. Rouzée, F. Lépine, S. Cohen, F. Robicheaux, A. Gijsbertsen, J. H. Jungmann, C. Bordas, and M. J. J. Vrakking, Phys. Rev. Lett. 110, 213001 (2013).

[6] C. Nicole, H. L. Offerhaus, M. J. J. Vrakking, F. Lépine, and C. Bordas, Phys. Rev. Lett. 88, 133001 (2002); C. Bordas, F. Lépine, C. Nicole, and M. J. J. Vrakking, Phys. Rev. A 68 , 012709 (2003); F. Lépine, C. Bordas, C. Nicole, and M. J. J. Vrakking, ibid. 70, 033417 (2004).
[7] S. Cohen, M. M. Harb, A. Ollagnier, F. Robicheaux, M. J. J. Vrakking, T. Barillot, F. Lépine, and C. Bordas, Phys. Rev. Lett. 110, 183001 (2013).

[8] S. Cohen, M. M. Harb, A. Ollagnier, F. Robicheaux, M. J. J. Vrakking, T. Barillot, F. Lépine, and C. Bordas, Phys. Rev. A 94, 013414 (2016).

[9] L. B. Zhao, I. I. Fabrikant, J. B. Delos, F. Lépine, S. Cohen and C. Bordas, Phys. Rev. A 85, 053421 (2012); L. B. Zhao, I. I. Fabrikant, M. L. Du, and C. Bordas, ibid. 86, 053413 (2012).

[10] L. B. Zhao, Europhys. Lett. 109, 23002 (2015).

[11] P. Giannakeas, F. Robicheaux, and C. H. Greene, Phys. Rev. A 91, 043424 (2015).

[12] F. Texier, Phys. Rev. A 71, 013403 (2005).

[13] A. S. Stodolna, F. Lépine, T. Bergeman, F. Robicheaux, A. Gijsbertsen, J. H. Jungmann, C. Bordas, and M. J. J. Vrakking, Phys. Rev. Lett. 113, 103002 (2014). 
[14] M. L. Zimmerman, M. G. Littman, M. M. Kash, and D. Kleppner, Phys. Rev. A 20, 2251 (1979); S. Feneuille, S. Liberman, E. Luc-Koenig, J. Pinard, and A. Taleb, J. Phys. B 15, 1205 (1982); J. Y. Liu, P. McNicholl, D. A. Harmin, J. Ivri, T. Bergeman, and H. J. Metcalf, Phys. Rev. Lett. 55, 189 (1985); P. McNicholl, T. Bergeman, and H. J. Metcalf, Phys. Rev. A 37, 3302 (1988).

[15] P. Kalaitzis, S. Danakas, F. Lépine, C. Bordas and S. Cohen, Phys. Rev. A 97, 053412 (2018).

[16] S. Ohgoda, O. I. Tolstikhin and T. Morishita, Phys. Rev. A 95, 043417 (2017).

[17] M. M. Harb, S. Cohen, E. Papalazarou, F. Lépine and C. Bordas, Rev. Sci. Instrum. 81, 125111 (2010).

[18] A. T. J. B. Eppink and D. H. Parker, Rev. Sci. Instrum. 68, 3477 (1997).
[19] W. E. Cooke and T. F. Gallagher, Phys. Rev. A 17, 1226 (1978); M. G. Littman, M. M. Kash, and D. Kleppner, Phys. Rev. Lett. 41, 103 (1978).

[20] T. F. Gallagher, L. M. Humphrey, W. E. Cooke, R. M. Hill, and S. A. Edelstein, Phys. Rev. A 16, 1098 (1977); J.-L. Vialle and H. T. Duong, J. Phys. B 12, 1407 (1979).

[21] J. I. Lo, C. C. Chu, H. S. Fung, Y. Y. Lee, and T. S. Yih, Chinese J. Phys. 51, 56 (2013).

[22] D. A. Harmin, Phys. Rev. A 24, 2491 (1981); 26, 2656 (1982).

[23] A. K. Kazansky, V. N. Ostrovsky and D. A. Telnov, J. Phys. B 23, L433 (1990).

[24] M. H. Rice and R. H. Good, J. Opt. Soc. Am. 52, 239 (1962).

[25] L. B. Zhao and J. B. Delos, Phys. Rev. A 81, 053418 (2010).

[26] S. Cohen, J. Phys. B 44, 205402 (2011). 